\title{
Analysis of the Effects of Phase-lags on Propagation of Harmonic Plane Waves in Thermoelastic Media
}

\author{
Roushan Kumar, Santwana Mukhopadhyay* \\ Department of Applied Mathematics, Institute of Technology, \\ Banaras Hindu University, Varanasi-221005, India \\ *Corresponding author: e-mail: mukho_santwana@rediffmail.com
}

(Received: 3 July 2009; revised: 16 December 2009; accepted: 30 December 2009; published online: 25 March 2010)

\begin{abstract}
The present paper attempts to investigate the propagation of harmonic plane waves of assigned frequency by employing the thermoelasticity theory with three phase-lags, recently proposed by Roychoudhuri (2007). The solutions of dispersion relation for the longitudinal plane waves are determined analytically and asymptotic expansions of several characterizations of the wave fields- phase velocity, specific loss and penetration depth of the dilatational waves are obtained for both the high frequency and low frequency values. Computational work for numerical values of the above quantities is also carried out with the help of Mathematica. A detailed analysis of the effects of phase-lags on the plane wave is presented by contrasting the theoretical as well as numerical results of the present work with the corresponding results of the theory of thermoelasticity type III (Green and Naghdi, 1993) as reported earlier.
\end{abstract}

Key words: harmonic plane wave, generalized thermoelasticity, thermoelasticity type III, thermoelasticity with three phase-lags

\section{INTRODUCTION}

The generalized theories of thermoelasticity have been developed to overcome the infinite propagation speed of thermal signals predicted by classical coupled dynamical theory of thermoelasticity (Biot [1]), the so-called "paradox of heat conduction". The subject of generalized thermoelasticity covers a wide range of extensions of classical theory of thermoelasticity. We recall the first two earliest and well-known generalized theories proposed by Lord and Shulman [2] and Green and Lindsay [3]. In the model [2], the Fourier law of heat conduction is replaced by MaxwellCattaneo law that introduces one thermal relaxation time parameter in the Fourier law whereas in the model of Green and Lindsay, two relaxation parameters are introduced in the constitutive relations for the stress tensor and the entropy. Later on, Green and Naghdi [4-6] proposed another three models, which are subsequently referred to as GN-I, II and III models. The linearized version of model-I corresponds to the classical thermoelastic model. In model-II the internal rate of production of entropy is taken to be identically zero implying no dissipation of thermal energy. This model admits undamped thermoelastic waves in a thermoelastic material and is best known as the theory of thermoelasticity without energy dissipation. Model-III includes the previous two models as special cases, and admits dissipation of energy in general. In this model, the proposed heat conduction law is of the form:

$$
\vec{q}(P, t)=-\left[K \vec{\nabla} \theta(P, t)+K^{*} \vec{\nabla} v(p, t)\right],
$$

where $\dot{v}=\theta$, and $\dot{q}, \theta, v$ being the heat flux vector, the temperature and the thermal displacement. Here both $K$ and $K^{*}$ are positive constants. $K$ is the thermal conductivity and $K^{*}$ is the conductivity rate. The $K^{*}$ (of physical dimension conductivity/time) is a material constant, characteristic of the theory. Subsequently, Tzou [7] proposed a dual phaselag heat conduction model to incorporate the effects of microscopic interactions in the fast-transient process of heat transport mechanism in a macroscopic formulation. Two different phase-lags (one for the heat flux vector and the other for the temperature gradient) have been introduced in the constitutive relation between heat flux vector and the temperature gradient. The law of heat conduction is thus generalized to the form

$$
\vec{q}\left(P, t+\tau_{q}\right)=-\left[k \vec{\nabla} \theta\left(P, t+\tau_{T}\right)\right] .
$$


The $\tau_{q}$ is interpreted as the relaxation time due to fast transient effects of thermal inertia, and $\tau_{T}$ is the phase-lag of temperature gradient, interpreted as the delay time caused by the microstructural interactions (small scale effects of heat transport in space such as phonon electron interaction or phonon scattering). Stability of dual-phase lag heat conduction was discussed by Quintanilla [8]. Thermoelastic model corresponding to dual-phase-lag heat conduction is proposed by Chandrasekharaiah [9]. Later on, Hetnarski and Ignaczak [10] introduced another model referred to as the low temperature thermoelastic model. In this model, unlike in the classical thermoelasticity theory, the heat flux and the free energy depend on the temperature, the strain tensor and the elastic heat flow. An extensive review on the development of the above-mentioned models is reported by Chandrasekharaiah [9]. Hetnarski and Ignaczak [11] also examined thoroughly the abovementioned models by focusing the theoretical significance of the models.

Very recently, Roychoudhuri [12] has introduced a new model of thermoelasticity theory, called the three-phase-lag thermoelastic model, in which the Fourier law is replaced by an approximation of the equation

$$
\vec{q}\left(P, t+\tau_{q}\right)=-\left[K \vec{\nabla} \theta\left(P, t+\tau_{T}\right)+K^{*} \vec{\nabla} v\left(p, t+\tau_{v}\right)\right]
$$

that includes three different phase-lags for the heat flux vector, the temperature gradient and the thermal displacement gradient. Here $\tau_{v}$, the delay time in thermal displacement gradient is also introduced in addition to $\tau_{q}$ and $\tau_{T}$. This model is more generalized and contains the previous models as special cases. The stability in the three phase-lag heat conduction equation and the relations among the three material parameters are discussed by Quintanilla and Racke [13]. Recently, Kar and Kanoria [14] employed this theory of thermoelasticity with three phase-lags to discuss a problem of thermoelastic interactions on functionally graded orthotropic hollow sphere under thermal shock. A problem of a spherical shell of viscoelastic medium of Kelvin-Voigt type is also studied by Kar and Kanoria [15] in the context of the thermoelasticity theory with three phase-lags.

Several researchers have attempted to investigate the propagation of harmonic plane waves in elastic medium. The propagation of plane waves in classical thermoelasticity is discussed by Lessen [16 ], Deresiewicz [17], Chadwick and Sneddon [18] and Chadwick [19]. Nayfeh and Nemat-Nasser [20] and Puri [21] studied the propagation of plane waves in generalized thermoelasticity with one relaxation time. The propagation and stability of harmonically time-dependent thermoelastic plane waves in
TRDTE is reported by Agarwal [22]. Wave propagation under Green-Lindsay model and Lord-Shulman model are re-examined by Haddow and Wegner [23]. Later on, Suh and Burger [24] found the order of magnitude of thermal relaxation time in TRDTE. Chandrasekharaiah [25] discussed the plane waves in the context of thermoelasticity of GN-II model. Puri and Jordan [26] investigated the propagation of plane waves under GN-III thermoelasticity theory.

The purpose of the present investigation is to study the propagation of plane harmonic waves in a homogeneous and isotropic unbounded medium in the context of the three phase-lag thermoelastic model. Firstly, we obtain the dispersion relation solutions of longitudinal plane waves and then we find the asymptotic expansions of several qualitative characterizations of the wave fields, such as phase velocity, specific loss and penetration depth for the high and low frequency values. Numerical computation of the above-mentioned quantities for intermediate values of frequency are carried out by using the software package Mathematica. In order to analyze the effects of phase-lags on the propagation of waves, a comparative analysis of the analytical and numerical results obtained in the present problem with those of GN-III model as reported in [26] is presented in a detailed way.

\section{GOVERNING EQUATIONS}

The field equations governing the displacement and the thermal fields in isotropic media in the absence of body forces are

$$
\begin{gathered}
\sigma_{i j, j}=\rho \ddot{u}_{i}, \\
\sigma_{i j}=\lambda e \delta_{i j}+2 \mu e_{i j}-\beta\left(\theta-\theta_{0}\right) \delta_{i j}, \\
e_{i j}=\frac{1}{2}\left(u_{i, j}+u_{j, i}\right) .
\end{gathered}
$$

The heat conduction equation in the context of hyperbolic thermoelasticity with three phase-lags [12] is

$$
\begin{gathered}
K^{*} \nabla^{2} \theta+\tau_{v}^{*} \nabla^{2} \dot{\theta}+K \tau_{T} \nabla^{2} \ddot{\theta}= \\
=\left(1+\tau_{q} \frac{\partial}{\partial t}+\frac{1}{2} \tau_{q}^{2} \frac{\partial^{2}}{\partial t^{2}}\right)\left(p c_{v} \ddot{\theta}+\beta T_{0} \ddot{u}_{i, i}\right),
\end{gathered}
$$

where $\tau_{v}^{*}=K+K^{*} \tau_{v}$.

In the above equations, $u_{i}$ is the component of displacement, $e_{i j}$ is the strain tensor, $\sigma_{i j}$ is the stress tensor, $\lambda$ . and $\mu$ are Lame's elastic constants, $\beta=(3 \lambda+2 \mu) \alpha_{t}$, $\alpha_{t}-$ coefficient of linear thermal expansion. $\theta$ is the absolute 
temperature, $T_{0}$ is the reference temperature, $\rho$ is the mass density, $c_{v}$ is the specific heat at constant strain. The $\tau_{T}, \tau_{q}$ and $\tau_{v}$ are the phase-lags of the temperature gradient, the heat flux and of thermal displacement gradient respectively, such that $\tau_{v}<\tau_{T}<\tau_{q} . K$ is the thermal conductivity. $K^{*}$ is the rate of thermal conductivity and characteristic of the theory of thermoelasticity of GN-III and the thermoelasticity with three phase-lags. The $\nabla^{2}$ is the Laplacian operator. A dot denotes the differentiation with respect to time $t$.

$e$ is the dilatation and given by

$$
e=e_{i i} \text {. }
$$

From Equations (1), (2), and (3) we obtain a displacement equation of motion as

$$
\mu u_{i, j j}+(\lambda+\mu) u_{j, j i}-\beta \theta_{,_{i}}-\rho \ddot{u}_{i} .
$$

Using the relation $\tau_{v}^{*}=K+K^{*} \tau_{v}$, the Equation (4) is reduced to the form

$$
\begin{gathered}
K^{*} \theta_{s_{i i}}+\left(K+K^{*} \tau_{v}\right) \dot{\theta}_{{ }_{i i}}+K \tau_{T} \ddot{\theta}_{, i i}= \\
=\left(1+\tau_{q} \frac{\partial}{\partial t}+\frac{1}{2} t_{q}^{2} \frac{\partial^{2}}{\partial t^{2}}\right)\left(\rho c_{v} \ddot{\theta}+\beta T_{0} \ddot{u}_{i, i}\right) .
\end{gathered}
$$

For the sake of simplicity, we introduce the non-dimensional variables and quantities

$$
\begin{gathered}
u_{i}^{\prime}=\frac{u_{i}}{c_{0} t_{r}}, \quad \theta^{\prime}=\frac{\theta-T_{0}}{T_{0}}, \quad x_{i}^{\prime}=\frac{x_{i}}{c_{0} t_{r}}, \\
t^{\prime}=\frac{t}{t_{r}}, \quad \tau_{v}^{\prime}=\frac{\tau_{v}}{t_{r}}, \quad \tau_{T}^{\prime}=\frac{\tau_{T}}{t_{r}}, \quad \tau_{q}^{\prime}=\frac{\tau_{q}}{t_{r}}, \\
K_{1}=\frac{K^{*}}{\rho c_{v} c_{0}^{2}}, \quad K_{2}=\frac{K}{\rho c_{v} c_{0}^{2} t_{r}}, \quad a_{2}=\frac{\beta}{\rho c_{v}},
\end{gathered}
$$

where $t_{r} \quad(>0)$ is a characteristic response time for a medium and $c_{0}=\sqrt{(\lambda+2 \mu) / \rho}$ is the velocity of longitudinal elastic wave. For the clarity in the equations of our analysis, we drop the primes from now onwards.

The Equations (5) and (6) therefore reduce to the forms

$$
\begin{aligned}
& \rho c_{0}^{2} \ddot{u}_{i}=\mu u_{i, j j}+(\lambda+\mu) u_{j, j i}-\beta T_{0} \theta_{, i} \\
& K_{1} \theta_{, i i}+\left(K_{2}+K_{1} \tau_{v}\right) \dot{\theta}_{, i i}+K_{2} \tau_{T} \ddot{\theta},_{i i}= \\
& =\left(1+\tau_{q} \frac{\partial}{\partial t}+\frac{1}{2} \tau_{q}^{2} \frac{\partial^{2}}{\partial t^{2}}\right)\left(\ddot{\theta}+a_{2} \ddot{u}_{i, i}\right) .
\end{aligned}
$$

The transverse waves can be shown to be not modified by, nor contributed to, the temperature field of the medium
[20]. Therefore, we will be seeking only the longitudinal plane wave solution in the form

$$
\begin{gathered}
u_{j}=A d_{j} e^{i\left(\omega t-\gamma n_{i} x_{i}\right)} \\
\theta=B e^{i\left(\omega t-\gamma n_{i} x_{i}\right)},
\end{gathered}
$$

where $\omega>0$ is the dimensionless frequency of wave, $d_{j}$ is the unit vector in the direction of displacement, $n_{j}$ is the unit vector normal to the wave front and $x_{j}$ is the position vector. $\gamma$ is a complex constant and $A$ and $B$ are complex amplitudes. $\omega / \operatorname{Re}(\gamma)$ is phase velocity of waves. Equations (9) and (10) then correspond to the dilatational waves for which $\omega / 2 \pi$ is the frequency and $2 \pi / \operatorname{Re}(\gamma)$ is the wavelength. Obviously, for the waves to be physically realistic $\operatorname{Re}(\gamma)>0$ and $\operatorname{Im}(\gamma) \leq 0$ must hold.

\section{DERIVATION OF DISPERSION RELATION}

Substituting Equations (9) and (10) into Equations (7) and (8) and using the property $d_{j} n_{j}=n_{j} n_{j}=1$, we obtain after some manipulations

$$
\left(\gamma^{2}-\omega^{2}\right) A-i a_{1} \gamma B=0
$$

$$
\begin{gathered}
\left(-a_{2} \tau_{q} \omega^{3} \gamma-i\left[a_{2} \omega^{2}-\frac{1}{2} \tau_{q}{ }^{2} \omega^{4}\right] \gamma\right) A+ \\
+B\left\{\gamma^{2}\left[K_{1}-\tau_{T} K_{2} \omega^{2}+i\left(\omega K_{2}+\omega K_{1} \tau_{v}\right)\right]+\right. \\
\left.-\omega^{2}+\frac{1}{2} \tau_{q}{ }^{2} \omega^{4}-i \tau_{q} \omega^{3}\right\}=0,
\end{gathered}
$$

where

$$
a_{1}=\frac{\beta T_{0}}{\lambda+2 \mu} .
$$

For non-trivial solutions, the determinant of the coefficient matrix in the above system of Equations (11) and (12) must be zero. Then we have a quadratic dispersion relation

$$
\begin{gathered}
\gamma^{4}\left(K_{1}-K_{2} \tau_{T} \omega^{2}+i\left(K_{2}+K_{1} \tau_{v}\right) \omega\right)+ \\
-\gamma^{2}\left[\left(K_{1}+h\right) \omega^{2}-\left(K_{2} \tau_{T}+\frac{\tau_{q}{ }^{2} h}{2}\right) \omega^{4}+\right. \\
\left.+i\left(K_{2}+\tau_{q} h+K_{1} \tau_{v}\right) \omega^{3}\right]+ \\
+\left(\omega^{4}-\frac{\tau_{q}{ }^{2} h \omega^{6}}{2}\right)+i \tau_{q} \omega^{5}=0
\end{gathered}
$$

where $\varepsilon=a_{1} a_{2}, h=1+\varepsilon$. 
By setting $Z=\gamma / \omega$ and multiplying throughout by $K_{1}-K_{2} \tau_{T} \omega^{2}-i\left(K_{2}+K_{1} \tau_{v}\right) \omega$ we arrive at

$$
\begin{gathered}
\left(\left(K_{1}\right)^{2}+A_{1} \omega^{2}+\left(K_{2}\right)^{2} \tau_{T}^{2} \omega^{4}\right) Z^{4}+ \\
-[P-i Q] Z^{2}+\left(K_{1}+A_{2} \omega^{2}+\frac{1}{2} K_{2} \tau_{q}{ }^{2} \tau_{T} \omega^{4}\right)+ \\
+i\left(-A_{3} \omega+A_{4} \omega^{3}\right)=0 .
\end{gathered}
$$

Here we have used the notations

$$
\begin{gathered}
P=N_{1}+N_{2} \omega^{2}+A_{5} \omega^{4}, \quad Q=h A_{3} \omega-h A_{4} \omega^{3}, \\
N_{1}=h K_{1}+\left(K_{1}\right)^{2}, \quad N_{2}=A_{1}+A_{2} h, \\
A_{1}=\left(K_{2}\right)^{2}-2 K_{1} K_{2} \tau_{T}+2 K_{1} K_{2} \tau_{v}+\left(K_{1}\right)^{2} \tau_{v}{ }^{2}, \\
A_{2}=K_{2} \tau_{q}-K_{2} \tau_{T}+K_{1} \tau_{q} \tau_{v}-\frac{1}{2} K_{1} \tau_{q}{ }^{2}, \\
A_{3}=K_{2}-K_{1} \tau_{q}+K_{1} \tau_{v}, A_{4}=\frac{1}{2} K_{2} \tau_{q}{ }^{2}+\frac{1}{2} K_{1} \tau_{q}{ }^{2} \tau_{v}-K_{2} \tau_{q} \tau_{T}, \\
A_{5}=K_{2} \tau_{T}\left(\frac{1}{2} h \tau_{q}{ }^{2}+K_{2} \tau_{T}\right) .
\end{gathered}
$$

The dispersion relation (14) clearly reflects the influence of the phase-lag parameters on the harmonic wave. It is noted here that for the special case when $\tau_{T}=\tau_{q}=\tau_{v}=0$, i.e. the case when all three phase-lags are zero, the Equation (14) reduces to the corresponding equation of GN-type III as reported by Puri and Jordan [26]. The dispersion relation (14) is therefore the more generalized one.

\section{EXPRESSIONS FOR ATTENUATION COEFFICIENTS AND WAVE NUMBER}

The roots of Equation (14) are $\pm Z_{1}$ and $\pm Z_{2}$ where

$$
\left(Z_{1,2}\right)^{2}=\frac{P-i Q \pm \sqrt{D(\omega)}}{2\left(\left(K_{1}\right)^{2}+A_{1} \omega^{2}+\left(K_{2}\right)^{2} \tau_{T}^{2} \omega^{4}\right)}
$$

with

$$
\begin{gathered}
\operatorname{Re}[D(\omega)]=L_{1}+L_{2} \omega^{2}+L_{3} \omega^{4}+L_{4} \omega^{6}+L_{5} \omega^{8}, \\
\operatorname{Im}[D(\omega)]=M_{1} \omega+M_{2} \omega^{3}+M_{3} \omega^{5}+M_{4} \omega^{7} .
\end{gathered}
$$

where we denote

$$
\begin{gathered}
L_{1}=\left(h^{2}\left(K_{1}\right)^{2}-4\left(K_{1}\right)^{3}+2 h\left(K_{1}\right)^{3}+\left(K_{1}\right)^{4}\right) L_{3}, \\
L_{2}=-4 K_{1} A_{1}+2 h K_{1} A_{1}+2\left(K_{1}\right)^{2} A_{1}+2 h^{2} K_{1} A_{2}-4\left(K_{1}\right)^{2} A_{2}+ \\
+2 h\left(K_{1}\right)^{2} A_{2}-h^{2}\left(A_{3}\right)^{2} \\
L_{3}=\left(A_{1}\right)^{2}-4 A_{1} A_{2}+2 h A_{1} A_{2}+h^{2}\left(A_{2}\right)^{2}+2 h^{2} A_{3} A_{4}+2 h K_{1} A_{5}+ \\
+2\left(K_{1}\right)^{2} A_{5}-2\left(K_{1}\right)^{2} K_{2} \tau_{q}{ }^{2} \tau_{T}+ \\
-4 K_{1}\left(K_{2}\right)^{2} \tau_{T}{ }^{2}, \\
L_{4}=-h^{2}\left(A_{4}\right)^{2}+2 A_{5} A_{1}+2 h A_{5} A_{1}-2 K_{2} A_{1} \tau_{q}{ }^{2} \tau_{T}-4\left(K_{2}\right)^{2} A_{2} \tau_{T}{ }^{2} \\
L_{5}=\left(A_{5}\right)^{2}-2\left(K_{2}\right)^{3} \tau_{q}{ }^{2} \tau_{T}{ }^{3}, \\
M_{3}=-4 A_{1} A_{4}+2 h A_{1} A_{4}+2 h^{2} A_{2} A_{4}-2 h A_{3} A_{5}+4\left(K_{2}\right)^{2} A_{3} \tau_{T}{ }^{2} \\
M_{4}=2 h A_{4} A_{5}-4\left(K_{2}\right)^{2} A_{4} \tau_{T}{ }^{2} . \\
M_{1}=-2 h^{2} K_{1} A_{3}+4\left(K_{1}\right)^{2} A_{3}-2 h\left(K_{1}\right)^{2} A_{3}, \\
+2 h A_{1} A_{3}-2 h A_{1} A_{3}-2 h^{2} A_{2} A_{3}+2 h^{2} K_{1} A_{4}-4\left(K_{1}\right)^{2} A_{4}+
\end{gathered}
$$

Now, only two of the four roots of $Z$ given by Equation (15) have the imaginary parts as negative. We are interested only in these two roots as only these roots yield the negative values of the decay coefficient, $\operatorname{Im}(\gamma)$. To obtain these roots, we use the following theorem of complex analysis [27]:

\section{Theorem}

If $L=x+i y$ and $p^{2}=L$, where $p$ is a complex number, the values of $p$ are given by

$$
p= \pm\left[\sqrt{\frac{|L|+x}{2}}+i \operatorname{sign}(y) \sqrt{\frac{|L|-x}{2}}\right] \text {, }
$$

where

$$
\operatorname{sign}(y)= \begin{cases}+1 & y \geq 0 \\ -1 & y<0\end{cases}
$$

The two values of $\gamma$ can therefore be obtained from Equation (15) by using the above theorem and the relation $\gamma=Z \omega$. These two values of $\gamma$ correspond to two modes of the dilatational wave. One of these waves is predominately elastic and the other is predominately thermal in 
nature. Let the value of $\gamma$ associated with the former one be denoted by $\gamma_{1}$ and the other one by $\gamma_{2}$.

The general analysis of waves based on the roots given by (15) is highly complicated. We therefore consider ourselves to the analysis of wave and the effects of the thermal relaxation parameter on both the waves in the special cases which correspond to the waves of small frequency and waves of high frequency.

\section{High-frequency asymptotic expansions}

We consider $\omega>>1$. Expanding the expressions for $Z_{1}$ from Equation (15) in powers of $\omega^{-1}$ and using the relation $\gamma=\omega Z$, we obtain after detailed and long calculations the high frequency asymptotic expansions for $\gamma_{1}$ and $\gamma_{2}$ as follows:

$$
\begin{aligned}
& \gamma_{1}=\sqrt{\frac{A_{5}+\sqrt{L_{5}}}{2}} \frac{\omega}{K_{2} \tau_{T}}\left\{1+\frac{1}{2\left(A_{5}+\sqrt{L_{5}}\right)} \times\right. \\
& \times\left[N_{2}+\frac{L_{4}}{2 \sqrt{L_{5}}}+\frac{M_{4}{ }^{2}}{8 L_{5} \sqrt{L_{5}}}-\frac{A_{1}\left(A_{5}+\sqrt{L_{5}}\right)}{\left(K_{2} \tau_{T}\right)^{2}}+\right. \\
& \left.-\frac{1}{4}\left(h A_{4}+\frac{M_{4}}{2 \sqrt{L_{5}}}\right)^{2} \frac{1}{A_{5}+\sqrt{L_{5}}}\right] \frac{1}{\omega^{2}}+ \\
& \left.-i\left(-h A_{4}-\frac{M_{4}}{2 \sqrt{L_{5}}}\right) \frac{1}{2\left(A_{5}+\sqrt{L_{5}}\right)} \frac{1}{\omega}\right\} \\
& (\omega \rightarrow \infty), \\
& \gamma_{2}=\sqrt{\frac{A_{5}-\sqrt{L_{5}}}{2}} \frac{\omega}{K_{2} \tau_{T}}\left\{1+\frac{1}{2\left(A_{5}-\sqrt{L_{5}}\right)} \times\right. \\
& \times\left[N_{2}-\frac{L_{4}}{2 \sqrt{L_{5}}}-\frac{M_{4}{ }^{2}}{8 L_{5} \sqrt{L_{5}}}-\frac{A_{1}\left(A_{5}-\sqrt{L_{5}}\right)}{\left(K_{2} \tau_{T}\right)^{2}}+\right. \\
& \left.-\frac{1}{4}\left(-h A_{4}+\frac{M_{4}}{2 \sqrt{L_{5}}}\right)^{2} \frac{1}{A_{5}-\sqrt{L_{5}}}\right] \frac{1}{\omega^{2}}+ \\
& \left.-i\left(-h A_{4}+\frac{M_{4}}{2 \sqrt{L_{5}}}\right) \frac{1}{2\left(A_{5}-\sqrt{L_{5}}\right)} \frac{1}{\omega}\right\} \text {, } \\
& (\omega \rightarrow \infty) .
\end{aligned}
$$

\section{Low-frequency asymptotic expansions}

We consider $\omega<<1$. Then we find for this case that

$$
\left.\begin{array}{c}
\gamma_{1}=\sqrt{\frac{N_{1}+\sqrt{L_{1}}}{2 K_{1}}} \omega \times \\
\times\left\{\frac { 1 } { 2 } \left[\frac{8 A_{1} L_{1} \sqrt{L_{1}}+8 h A_{2} L_{1} \sqrt{L_{1}}+4 L_{1} L_{2}+M_{1}^{2}}{8 L_{1} \sqrt{L_{1}}\left(N_{1}+\sqrt{L_{1}}\right)}-\frac{A_{1}}{K_{1}}+\right.\right. \\
\left.-\frac{1}{4}\left(\frac{2 h A_{3} \sqrt{L_{1}}-M_{1}}{2 \sqrt{L_{1}}\left(N_{1}+\sqrt{L_{1}}\right)}\right)^{2}\right] \omega^{2}-i\left(\frac{2 h A_{1} A_{3} \sqrt{L_{1}}-M_{1}}{4 \sqrt{L_{1}}\left(N_{1}+\sqrt{L_{1}}\right)}\right) \omega
\end{array}\right\},
$$

It can be easily shown that all of the expressions: $\sqrt{L_{5}}$, $\sqrt{L_{1}}, A_{5} \pm \sqrt{L_{5}}$ and $N_{1} \pm \sqrt{L_{1}}$ are always positive.

\section{ANALYTICAL RESULTS}

To study the plane waves in details, we derive the asymptotic expressions of the important characterizations of wave field such as phase velocity, specific loss, penetration depth of both the modified elastic and modified thermal waves and examine these quantities under the cases of high frequency values as well as low-frequency values.

\section{(1) Phase velocity:}

The phase velocity is given by

$$
V_{E, T}=V_{1,2}=\frac{\omega}{\operatorname{Re}\left[\gamma_{1,2}\right]}
$$

where $V_{E}$ is the velocity of elastic wave and $V_{T}$ is the velocity of the thermal wave.

From Equation (16-19) and using (20) the high frequency and low frequency asymptotes are given by 


\section{High-frequency asymptotics}

$$
\begin{gathered}
V_{1}=\frac{\sqrt{2} K_{2} \tau_{T}}{\sqrt{\sqrt{L_{5}}+A_{5}}}+O\left(\frac{1}{\omega^{2}}\right) \quad(\omega \rightarrow \infty), \\
V_{2}=\frac{\sqrt{2} K_{2} \tau_{T}}{\sqrt{-\sqrt{L_{5}}+A_{5}}}+O\left(\frac{1}{\omega^{2}}\right) \quad(\omega \rightarrow \infty) .
\end{gathered}
$$

\section{Low-frequency asymptotics}

$$
\begin{aligned}
& V_{1}=\sqrt{\frac{2 K_{1}}{\sqrt{L_{1}}+N_{1}}}+O\left(\omega^{2}\right) \quad(\omega \rightarrow 0) \\
& V_{2}=\sqrt{\frac{2 K_{1}}{-\sqrt{L_{1}}+N_{1}}}+O\left(\omega^{2}\right) \quad(\omega \rightarrow 0)
\end{aligned}
$$

\section{(II) Specific loss:}

The specific loss, which is defended as the ratio of energy dissipated per stress cycle to the total vibrational energy is given by

$$
\left(\frac{\Delta W}{W}\right)_{E, T}=\left(\frac{\Delta W}{W}\right)_{1,2}=4 \pi\left|\frac{\operatorname{Im}\left[\gamma_{1,2}\right]}{\operatorname{Re}\left[\gamma_{1,2}\right]}\right| .
$$

From Equation (16-19) and (25) the high frequency and low frequency asymptotics are obtained as

\section{High-frequency asymptotics}

$$
\begin{aligned}
& \left(\frac{\Delta \mathrm{W}}{W}\right)_{1} \approx \frac{\left(-2 A_{4} h \sqrt{L_{5}}-M_{4}\right) \pi}{\sqrt{L_{5}}\left(\sqrt{L_{5}}+A_{5}\right) \omega}+O\left(\frac{1}{\omega^{3}}\right) \quad(\omega \rightarrow \infty) \\
& \left(\frac{\Delta \mathrm{W}}{W}\right)_{2} \approx \frac{\left(2 A_{4} h \sqrt{L_{5}}-M_{4}\right) \pi}{\sqrt{L_{5}}\left(\sqrt{L_{5}}-A_{5}\right) \omega}+O\left(\frac{1}{\omega^{3}}\right) \quad(\omega \rightarrow \infty) .
\end{aligned}
$$

\section{Low-frequency asymptotics}

$$
\begin{aligned}
& \left(\frac{\Delta \mathrm{W}}{W}\right)_{1} \approx \frac{\left(2 A_{3} h \sqrt{L_{1}}-M_{1}\right) \pi}{\sqrt{L_{1}}\left(\sqrt{L_{1}}+N_{1}\right)} \omega+O\left(\omega^{3}\right) \quad(\omega \rightarrow 0), \\
& \left(\frac{\Delta W}{W}\right)_{2}=\frac{\left(2 A_{3} h \sqrt{L_{1}}+M_{1}\right) \pi}{\sqrt{L_{1}}\left(-\sqrt{L_{1}}+N_{1}\right)} \omega+O\left(\omega^{3}\right) \quad(\omega \rightarrow 0) .
\end{aligned}
$$

\section{(III) Penetration depth:}

The Penetration depth is defined by

$$
\delta_{E, T}=\delta_{1,2}=\left|\frac{1}{\operatorname{Im}\left[\gamma_{1,2}\right]}\right|
$$

From Equation (16-19) and (30) we find the high frequency and low frequency asymptotics for penetration depth as

\section{High-frequency asymptotics}

$$
\begin{gathered}
\delta_{1} \approx \frac{4 \sqrt{2} K_{2} \sqrt{L_{5}} \sqrt{\sqrt{L_{5}}+A_{5}} \tau_{T}}{-\left(2 A_{4} h \sqrt{L_{5}}+M_{4}\right)}+O\left(\frac{1}{\omega^{2}}\right) \\
(\omega \rightarrow \infty), \\
\delta_{2} \approx \frac{2 \sqrt{2} K_{2} \sqrt{L_{5}} \sqrt{\sqrt{L_{5}}+A_{5}} \tau_{T}}{-2 A_{4} h \sqrt{L_{5}}+M_{4}}+O\left(\frac{1}{\omega^{2}}\right) \\
(\omega \rightarrow \infty) .
\end{gathered}
$$

\section{Low-frequency asymptotics}

$$
\begin{gathered}
\delta_{1} \approx \frac{4 \sqrt{2 K_{1} L_{1}} \sqrt{\sqrt{L_{1}}+N_{1}}}{\left(2 A_{3} h \sqrt{L_{1}}-M_{1}\right) \omega^{2}}+O\left(\omega^{3}\right) \quad(\omega \rightarrow 0), \\
\delta_{2}=\frac{4 \sqrt{2 K_{1} L_{1}} \sqrt{-\sqrt{L_{1}}+N_{1}}}{\left(2 A_{3} h \sqrt{L_{1}}+M_{1}\right) \omega^{2}}+O\left(\omega^{3}\right) \quad(\omega \rightarrow 0) .
\end{gathered}
$$

\section{NUMERICAL RESULTS}

In this section, we carry out the computational work in order to illustrate the asymptotic results derived in the previous section and thoroughly examine the behaviors of phase velocity, penetration depth and specific loss of waves. For this, we assume the non-dimensional values of the parameters as [26]

$$
\varepsilon=0.0168, K_{1}=1.0516, K_{2}=1 .
$$

We take $\tau_{v}=0.01, \tau_{T}=0.015, \tau_{q}=0.02$.

By employing software package, Mathematica 6.0 and using the formulae given in Equations (20), (25), and (30), we obtain the numerical values of the quantities for both the elastic and thermal mode waves directly from Equation (14) for various values of $\omega$. In order to analyze the effects of phase-lags we compare our results with the corresponding results of GN-III as reported by Puri and Jordan [26]. 
For this, we also compute the numerical values of different characterizations by putting $\tau_{T}=\tau_{q}=\tau_{v}=0$. The results are displayed graphically. In all the figures, the solid lines represent the profiles in case of thermoelasticity with three phase-lags (TETPL) model and the dashed lines represent the values in GN-III model.

\section{ANALYSIS OF ANALYTICAL AND NUMERICAL RESULTS}

\section{Phase velocity profile}

Figures 1(a) and 1(b) display the variation of phase velocity of an elastic mode wave with respect to $\omega$. Particularly, Figure 1(b) shows the variation of phase velocity for higher values of $\omega$. We observe from Fig. 1(a) that for smaller values of frequency the phase velocity of elastic mode wave is an increasing function of $\omega$ under both the models. The trend of variation is also similar under both the cases. However, Figure 1(b) reveals a prominent difference between the phase velocity profiles under TETPL and GN-III for higher values of frequency. In the case of TETPL, the phase velocity of elastic mode wave first increases to a maximum value and then decreases rapidly and approaches to its limiting value 0.999887 , whereas in case of GN-III model the phase velocity increases and approaches to its limiting value 1 . This is in complete agreement with our theoretical result (see expression (21)) and the corresponding result as reported by Puri and Jordan [26] which show that phase velocity of elastic wave approaches to its constant limiting value as $\omega \rightarrow \infty$ like $\sim \omega^{-2}$.

Figures $1(\mathrm{c}, \mathrm{d})$ represent the variation of phase velocity of a thermal mode wave with frequency $\omega$. We see from Fig. 1(c) that for smaller frequencies the phase velocity of a thermal wave is also an increasing function of $\omega$ and the nature of variation is similar under both the theories. However, for higher values of frequency a significant difference between two models is observed from Fig. 1(d). Under the case of TETPL, the phase velocity of the thermal mode wave increases and attains the limiting value 8.66124. This lends strong support to our theoretical predictions obtained from Equation (22) which shows that at infinity it approaches to the constant limiting value as $(\omega \rightarrow \infty)$. On the contrary, the phase velocity of the thermal mode wave is noticed to be an increasing function of $\omega$ in case of the GN-III model. This is also in agreement with the corresponding result as reported in [26] that it varies like $\sim \sqrt{\omega}$ as $(\omega \rightarrow \infty)$ in this case.

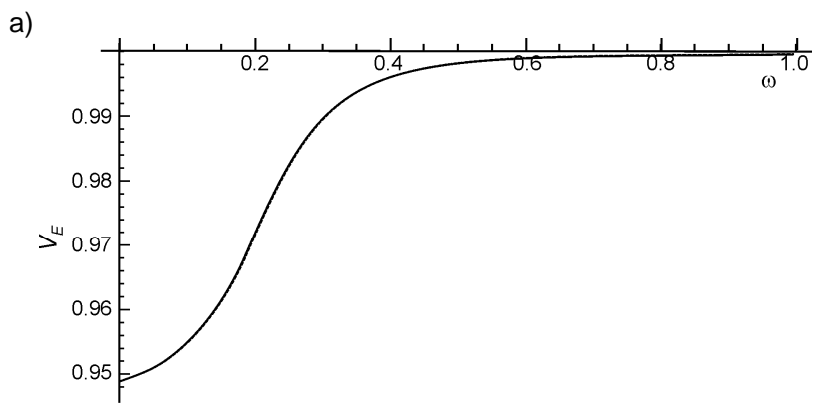

b)

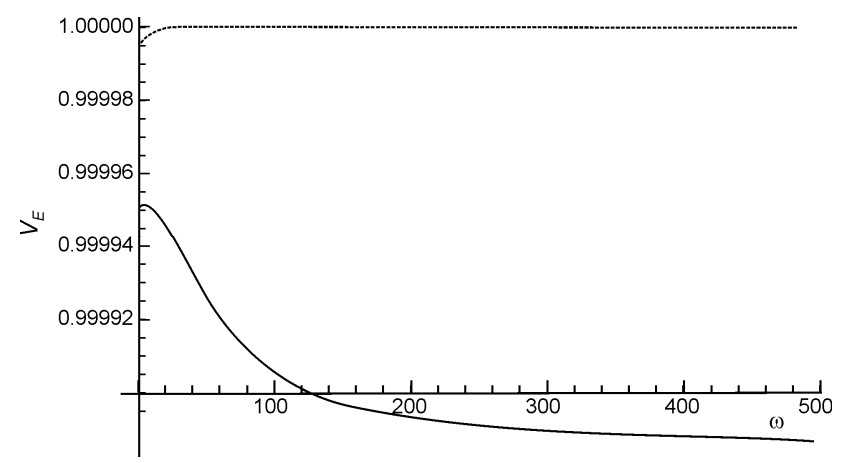

Fig. 1(a,b). Phase velocity of elastic mode wave: solid line: TETPL model, dashed line: GN-III model

a)

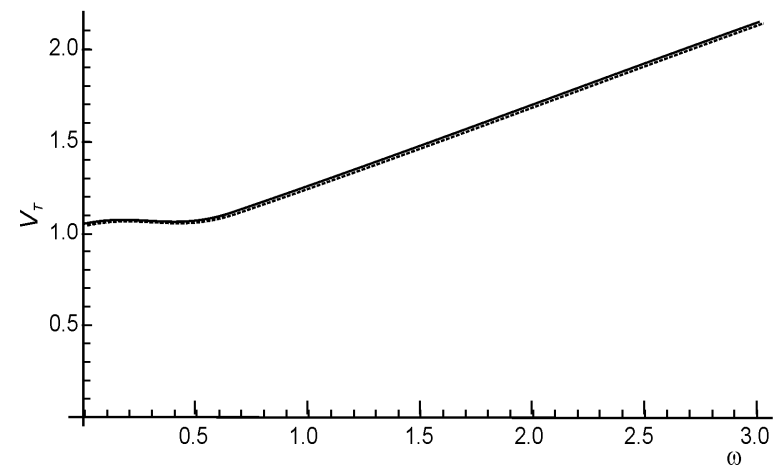

b)

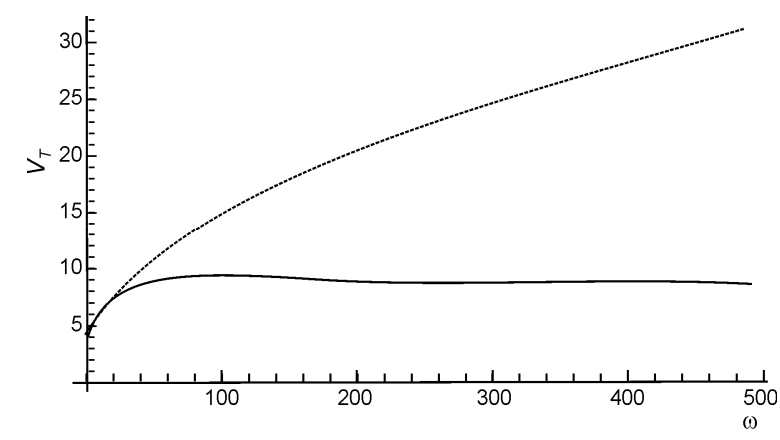

Fig. 1(c,d). Phase velocity of thermal mode wave: solid line: TETPL model, dashed line: GN-III model 


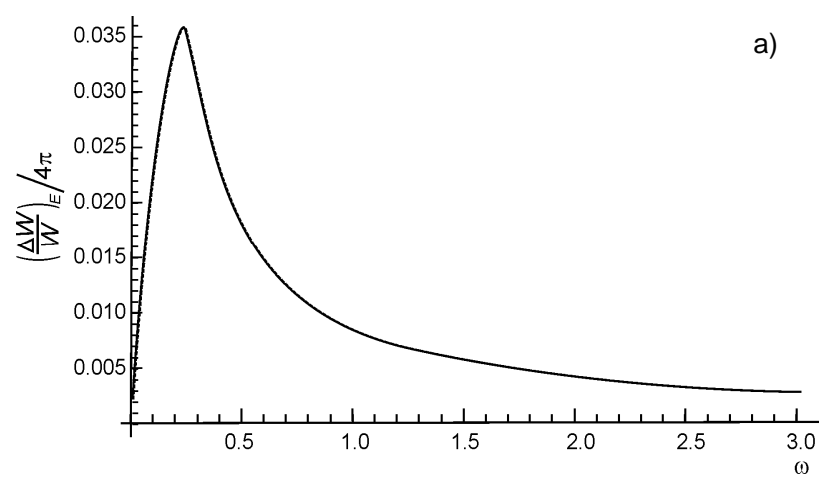

b)

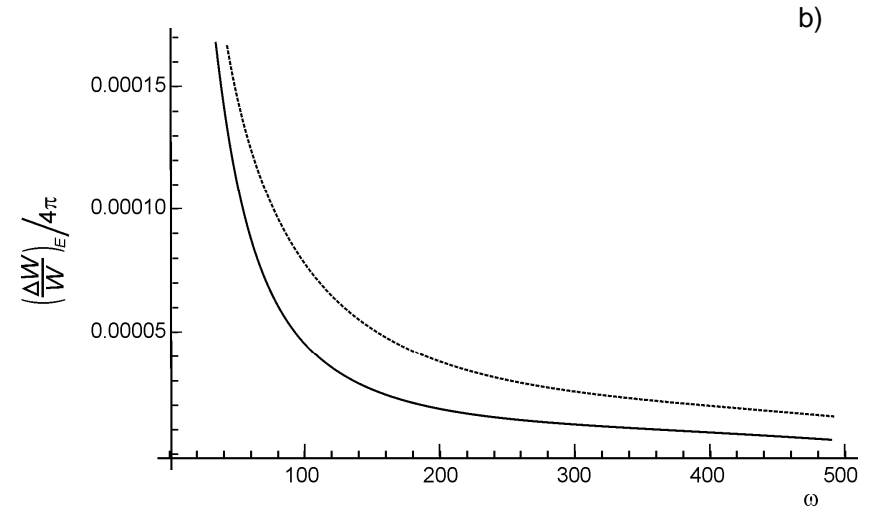

Fig. 2(a,b). Specific loss of elastic mode wave: solid line: TETPL model, dashed line: GN-III model

a)

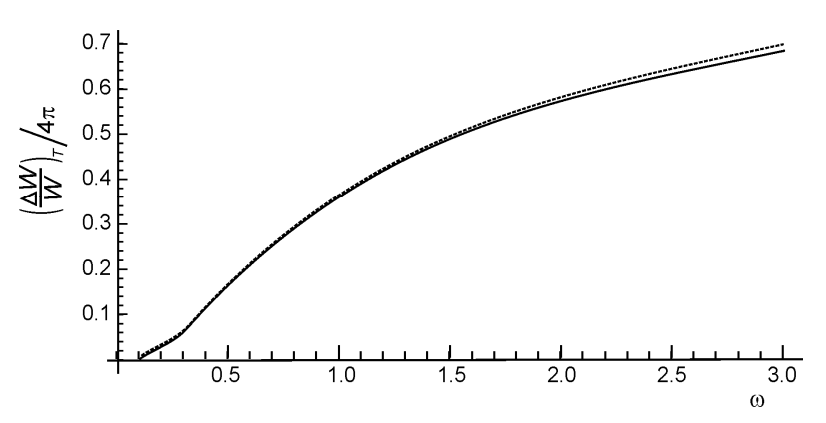

b)

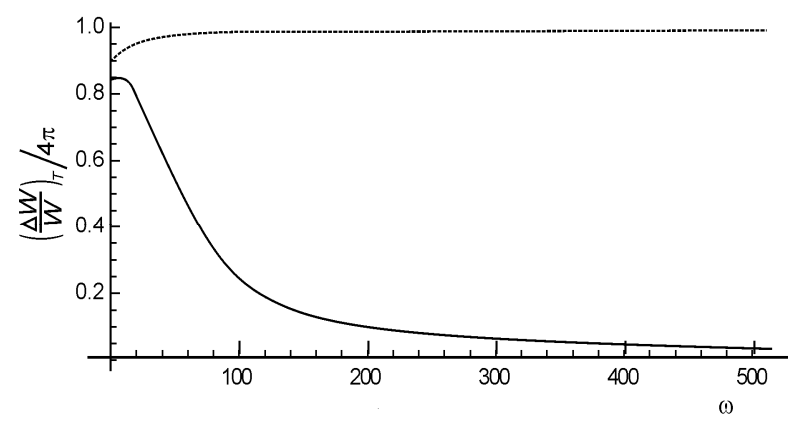

Fig. 2(c,d). Specific loss of thermal mode wave: solid line: TETPL model, dashed line: GN-III model



b)

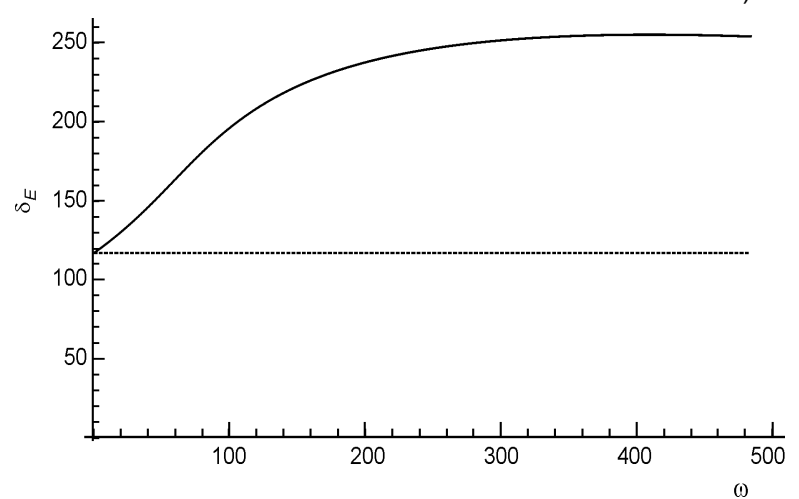

Fig. 3(a,b). Penetration depth of elastic mode wave: solid line: TETPL model, dashed line: GN-III model

a)

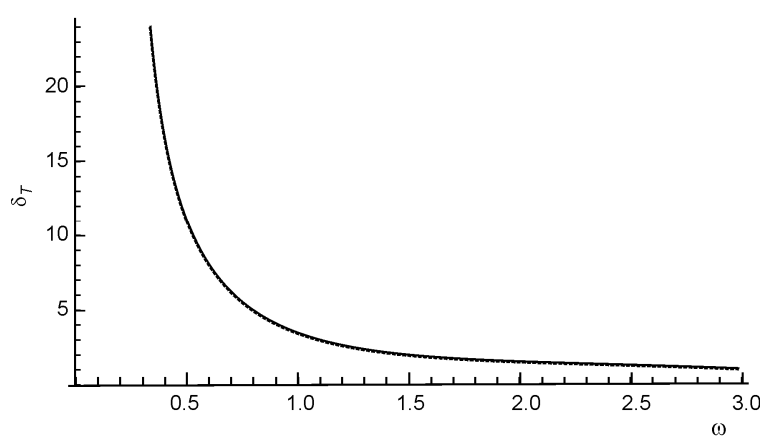

b)

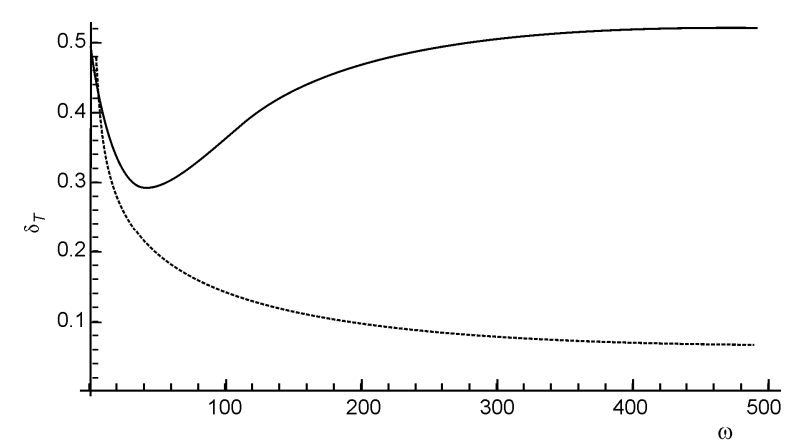

Fig. 3(c,d). Penetration depth of thermal mode wave: solid line: TETPL model, dashed line: GN-III model 


\section{Specific loss profile}

From Figures $2(\mathrm{a}, \mathrm{b})$ it is observed that under both the cases (GN-III and TETPL) the specific loss profile for the elastic mode wave also has similar behavior for smaller values of frequency by showing a stationary maximum and finally decreasing to zero. This profile shows a similar trend of variation under both the models for higher values of frequency, too. It approaches to zero as $(\omega \rightarrow \infty)$ under two different models. This agrees with the theoretical predictions as we observe from Equation (26) and the corresponding equation in [26] that under both the cases the specific loss approaches to zero value likes $\sim \omega^{-1}$ as $\omega \rightarrow \infty$.

The behavior of specific loss of the thermal mode wave is depicted in Fig. 2(c,d). Under both the models, specific loss of the thermal mode wave tends to zero value as $\omega \rightarrow 0$ and Fig. 2(c) shows that there exists no significant difference between two models for smaller frequencies. However, as $\omega$ increases, a disagreement by two models for this field is indicated in Fig. 2(d) as well as in the corresponding theoretical results (see Eq. (27) and Eq. (3.9) of [26]). We note that for higher values of frequency, this field continuously increases and approaches to its limiting value 1 in case of GNIII model, whereas it first increases from zero value, reaches to a maximum value and thereafter it starts decreasing and approaches to zero value as $\omega \rightarrow \infty$ in case of TETPL.

\section{Penetration depth profile}

Figures 3(a) and 3(c) show the variation of the penetration depth profile of the elastic wave and thermal wave respectively for smaller frequencies, and show that under both the models, the curves of this profile also have the similar trend. Penetration depth of elastic wave approaches to a constant limiting value as $\omega \rightarrow \infty$ under both the cases, although the numerical values under two cases are significantly different for higher frequencies (see Fig. 3(b)). The variation of the penetration depth profile for higher values of $\omega$ due to the thermal wave is depicted in Fig. 3(d). From this figure we note that as $\omega \rightarrow \infty$, it approaches to a limiting value (0.530961) in TETPL but in case of GN-III it decreases and finds its limiting value zero. These results are also completely in agreement with our theoretical results (Eqs. (31-34) and the results as reported by Puri and Jordan [26]).

\section{SUMMARY AND OBSERVATIONS}

Dispersion relation solutions are determined, and high and low-frequency asymptotic results are presented for the propagation of the harmonic plane wave propagating in a thermoelastic media by employing the theory of thermoelasticity with three phase-lags. The effects of phase-lags on propagation of wave are analyzed by comparing our results with those of thermoelasticity of type GN-III. We observed the following significant facts which reflect the influence of phase-lags on the plane waves:

(1) Like GN-III case, there are elastic and thermal mode dilatational wave corresponding to $\gamma_{1,2}$, for all $\omega>0$.

(2) For small frequency, a similar nature of the curves for characterizations of the wave fields like phase velocity, specific loss and penetration depth are observed for both the models.

(3) The effects of phase-lags on all characterizations are significant for higher values of frequency.

(4) As $\omega \rightarrow \infty$, all characterizations of the wave fields approach a limiting value in TETPL.

(5) For high frequency, the penetration depth profile shows higher values for both the elastic and thermal mode waves under TETPL.

\section{Acknowledgement}

We are grateful to the reviewers for their critical and constructive comments and suggestions, which have helped to improve the quality of our paper.

\section{References}

[1] M. Biot, Thermoelasticity and Irreversible thermodynamics. J. Appl. Phys. 27, 240-253 (1956).

[2] H.W. Lord and Y. Shulman, Generalized dynamical theory of thermoelasticity, J. Mech. Phys. Solids 15, 299-309 (1967).

[3] A.E Green, K.A Lindsay, Thermoelasticity. Journal of Elasticity 2, 1-7 (1972).

[4] A.E. Green, P.M. Naghdi, A re-examination of the basic postulates of thermoemechanics. Proc. Roy. Soc. London, A432, 171-194 (1991).

[5] A.E. Green, P.M. Naghdi, On undamped heat waves in an elastic solid. J. Thermal stresses 15, 253-264 (1992).

[6] A.E. Green, P.M. Naghdi, Thermoelasticity without energy dissipation. J. Elasticity 31, 189-209 (1993).

[7] D.Y. Tzou, A unified filed approach for heat conduction from Macro to Micro scales. ASME Journal of Heat transfer 117, 8-16 (1995).

[8] R. Quintanilla, R. Racke, A note on stability in dual-phaselag heat conduction, Int. J. Heat and Mass Transfer 49, 7-8, 1209-1213 (2006).

[9] D.S. Chandrasekharaiah, Hyperbolic thermoelasticity: A Review of recent literature. Appl. Mech. Rev. 39 (1998).

[10] R.B. Hetnarski, J. Ignaczak, Soliton-like waves in a low temperature and non linear thermoelastic solid. Int. J. Eng. Sci. 34, 1767-1787 (1996). 
[11] R. B. Hetnarski, J. Ignaczak, Generalized thermoelasticity. J. Thermal stresses 22, 451-476 (1999).

[12] S.K. Roychoudhuri, On a thermoelastic three-phase-lag model. J. Thermal Stresses 30, 231-238 (2007).

[13] R. Quintanilla, R. Racke, A note on stability in three-phaselag heat conduction. Int. J. Heat and Mass Transfer 51, 24-29 (2008).

[14] A. Kar, M. Kanoria, Generalized thermoelastic functionally graded orthotropic hollow sphere under thermal shock with three-phase-lag effect. European Journal of Mechanics A/Solids 28, 757-767 (2009).

[15] A. Kar, M. Kanoria, Generalized thermo-visco-elastic problem of a spherical shell with three-phase-lag effect, Appl. Math. Modelling 33, 3287-3298 (2009).

[16] M. Lessen, Motion of thermoelastic solid, Q. Appl. Maths. 15, 105-108 (1957).

[17] H. Deresiewicz, Plane waves in a thermoelastic solid, J. Acoust. Soc. Am. 29, 204-209 (1957).

[18] P. Chadwick, I.N. Sneddon, Plane waves in an elastic solid conducting heat. J. Mech. Phys. Solids 6, 223-230 (1958).
[19] P. Chadwick, Thermoelasticity: the dynamic theory, in: R. Hill, I.N. Sneddon (Eds.), Progress in Solid Mechanics, vol. I, North-Holland, Amsterdam 263-328 (1960).

[20] A. Nayfeh, S. Nemat-Nasser, Thermoelastic waves in solids with thermal Relaxation. Acta Mech.12, 53-69 (1971).

[21] P. Puri, Plane waves in generalized thermoelasticity, Int. J. Eng. Sci. 11, 735-744 (1973).

[22] V.K. Agarwal, On plane waves in generalized thermoelasticity. Acta Mech. 31, 185-198 (1979)

[23] J.B. Haddow, J.L. Wegner, Plane harmonic waves for three thermoelastic theories. Math. Mech. Solids 1, 111-127, (1996).

[24] C.S. Suh, C.P. Burger, Effects of thermomechanical coupling and relaxation times on wave spectrum in dynamic theory of generalized thermoelasticity. J. Appl. Mech. (Trans. ASME) 65, 605-613 (1998).

[25] D.S. Chandrasekharaiah, Thermoelastic plane waves without energy dissipation. Mech. Res. Commun. 23, 549-555 (1996).

[26] P. Puri, P.M. Jordan, On the propagation of plane waves in type-III thermoelastic media. Proc. R. Soc. A 460, 32033221, 2004.

[27] S. Punnusamy, Foundation of complex analysis. Narosa Publishing House (2001).

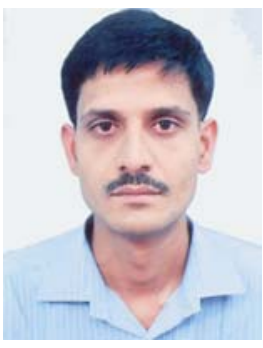

Mr. Roushan Kumar, 16 August, 1977, M.Sc in Mathematics, Banaras Hindu University, India, 2004; Nationality: Indian; E-mail: rkumar.rs.apm@itbhu.ac.in; Current affiliation: Ph.D Student, Institute of Technology, Banaras Hindu University, India; Professional: Theory of Thermoelasticity, Publications: 7 papers in peer reviewed Journals and 2 conference presentations.



Dr. Santwana Mukhopadhyay, 7 January, 1965, Ph.D in Applied Mathematics, University of Burdwan, India, 1998; Nationality: Indian; E-mail: mukhosant.apm@itbhu.ac.in; Membership: Mathematical Society, BHU; Current affiliation: Reader, Institute of Technology, Banaras Hindu University, India; Professional: Theory of Thermoelasticity, Computational Mathematics; Publications: 30 papers in peer reviewed Journals and 5 conference presentations; Honours: Awarded University Gold medal. 